\title{
“CATCH IT RIGHT"-A CASE REPORT ON OCCLUSAL SPLINT
}

\author{
Monika Makkar ${ }^{1}$, Poonam Pathania ${ }^{2}$, Swati Sharma ${ }^{3}$ \\ ${ }^{1}$ Professor, Department of Prosthodontics, Swami Devi Dyal Dental College and Hospital, Haryana, India \\ ${ }^{2}$ Post graduate student, SDDHC, Haryana, India \\ ${ }^{3}$ Post graduate student, SDDHC, Haryana, India
}

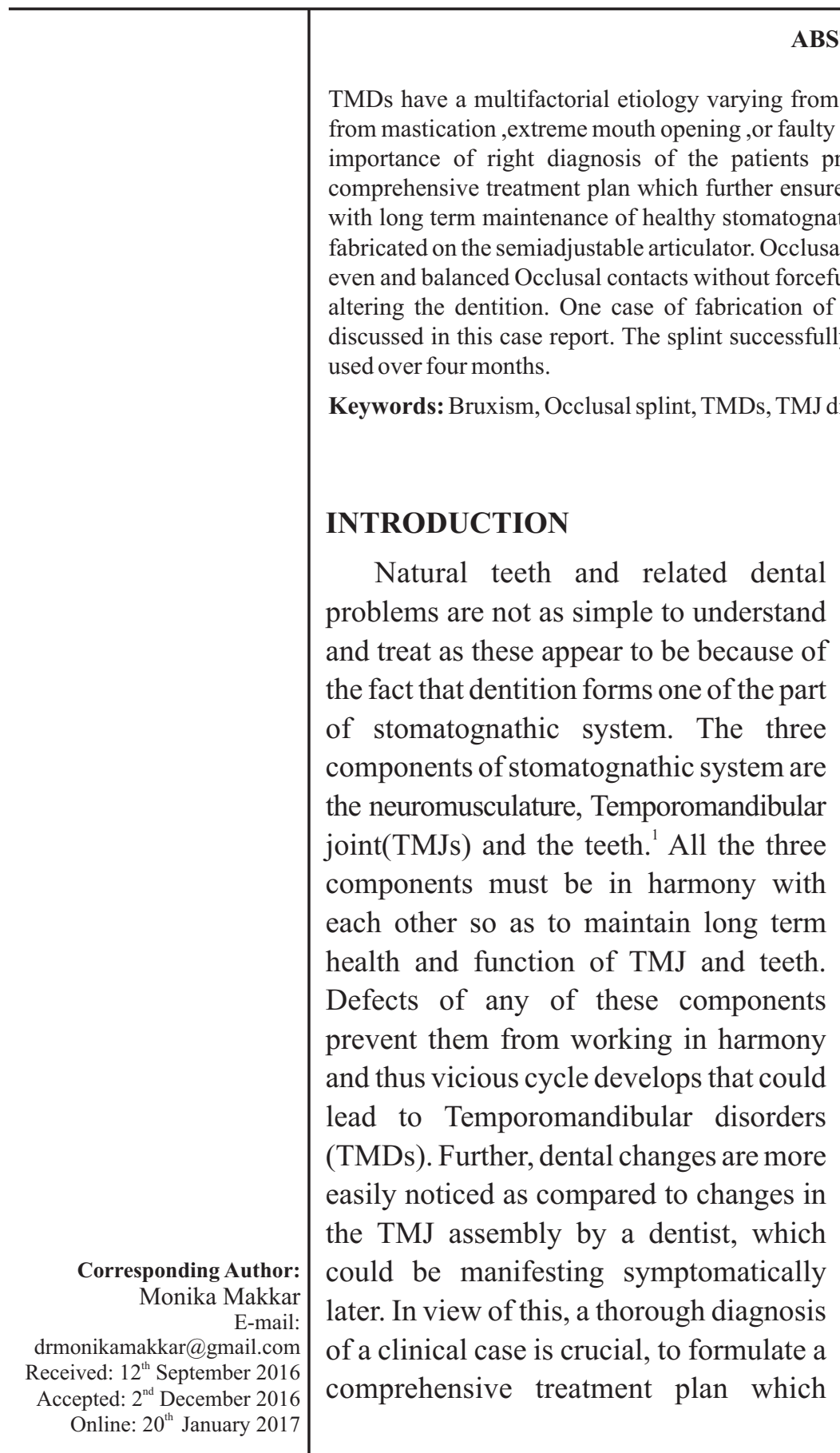

ensures the solving of the stated patient problem along with long term maintenance of healthy stomatognathic system.

TMDs have a multifactorial etiology varying from bruxism, psychological illness and traumatic injuries from mastication, due to extreme mouth opening, or faulty dental restorations. TMDs are characterized by clicking and pain, either confined to the TMJ region or radiating to the eyes, shoulder, and neck. Headaches, tinnitus, jaw deviation, locking, and limited mouth opening are common symptoms. Pain is the most crucial symptom for which patients seek medical care. Management of TMD includes conservative and surgical interventions. Conservative treatments are physical therapy, localized steam application, external muscle massage, occlusal adjustment, analgesia, psychotropic medication, splint therapy, 
alternative therapies such as acupuncture, as well as treatment modalities such as ultrasound, soft laser, diathermy, and infrared radiation.

\section{Case Report}

A 43yrs old male patient named Ravinder Kumar reported in the department of Prosthodontics, crown and bridge and oral implantology with a chief complaint of moderately severe pain on the right side of his lower jaw and mild pain on left side, since three to four months. History of present illness revealed the pain was less at rest and increased while eating routine food, yawning. The pain was sharp with feeling of jaw deviation towards right side and backwards. The patient discontinued eating hard food since then and started taking analgesics for a week as was prescribed and reported back with no relief in pain. Clicking in right TMJ was present on opening the mouth. The patient reported the habit of clenching and grinding his teeth while in anger.

Medical History revealed that patient is a controlled diabetic and was on medication since two years. The patient had cardiovascular problem three and a half years ago. The patient suffered from breathlessness and swelling in joints which was relieved after medication. The patient was first diagnosed of hyperthyroidism but later it was negated after tests. Patient was taking muscle relaxants and analgesics for TMJ pain. There was a history of emotional stress as well as habits of clenching and bruxism during driving and at night.

Extraoral examination revealed bilateral massetric hypertrophy along with the loss of vertical dimension (Figure 1). The palpation of TMJ revealed tenderness, more so on right side. There was pain especially in right TMJ, while opening of the mouth wide. Clicking was present on both sides. No deviation of movement was seen while opening of the mouth. On muscle examination, right side elevator muscles were found

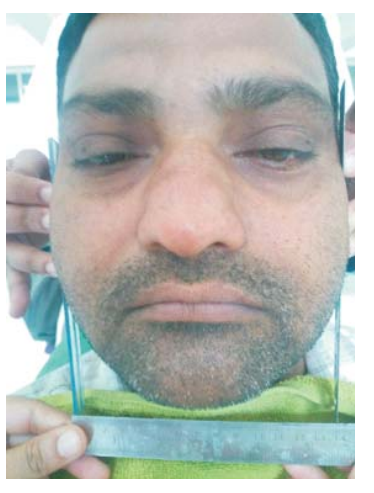

Figure 1: Bilateral Massetric muscle hypertrophy more tender as compared to left side. Right and left lateral pterygoids were found to be tender on intraoral palpation. Detailed evaluation of TMJ was done by using Fonseca's Questionnaire which gave score of 70 (details of the questionnaire ahead in the text). Dental examination showed generalized attrition of teeth (Figure 3,4) with loss of anterior guidance .Occlusal examination showed the posterior teeth contacts in protrusive mandibular movements (Figure 5) and there were contacts present on non working side during lateral excursions (Figure 6).

Dental orthopantanogram of the patient (Figure 2), showed bilateral subcondylar sclerosis. Bilateral Spruing of angle of mandible, suggested of massesteric hyperactivity.

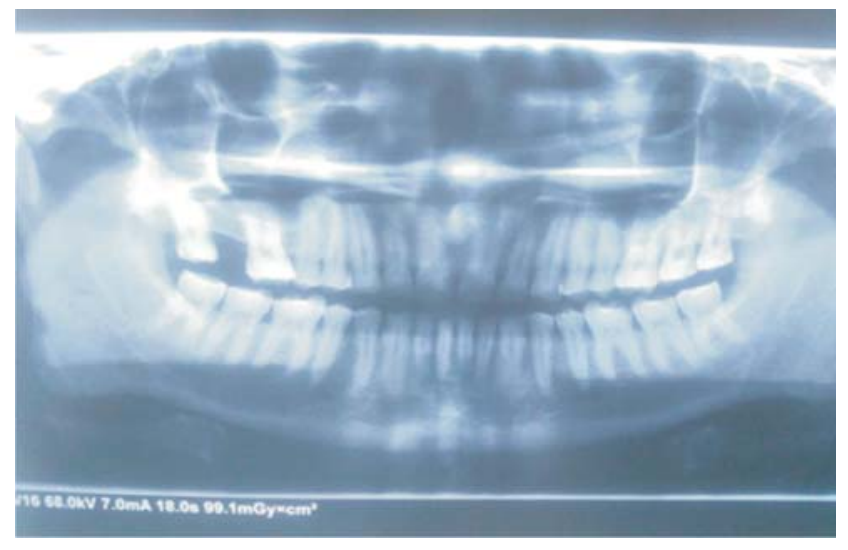

Figure 2: Orthopantanogram of the patient

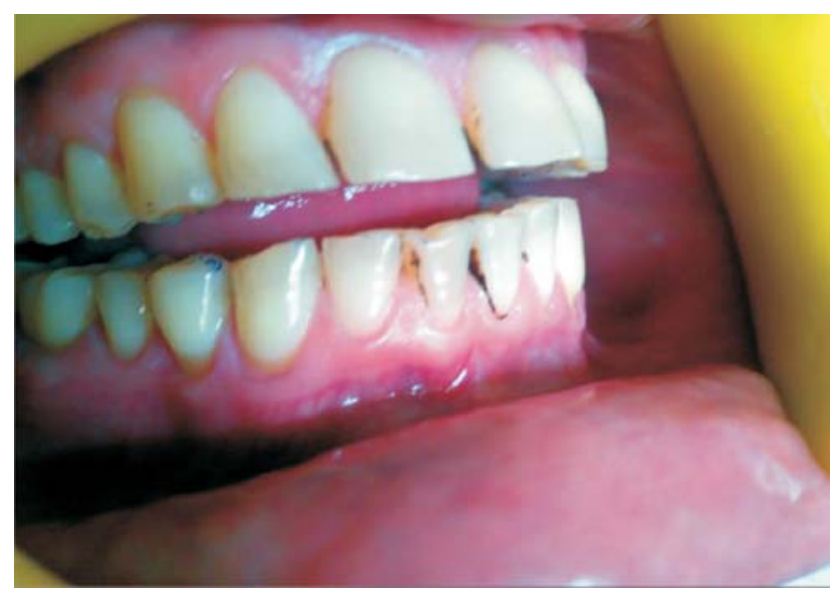

Figure 3: Incisal and occlusal wear

Provisional Diagnosis of occlusal instability leading to TMD along with muscle hyperactivity due to clenching or bruxism was extracted. 


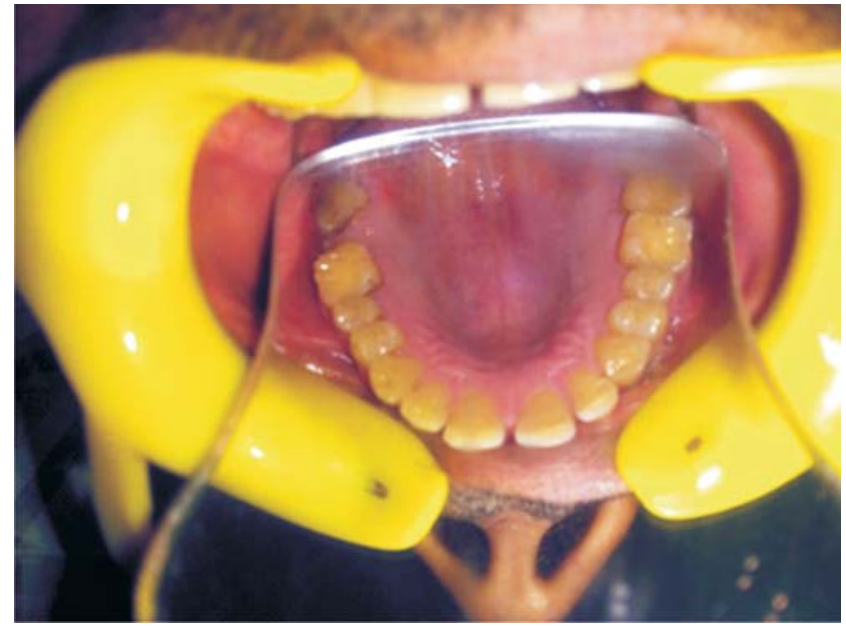

Figure 4: Occlusal wear

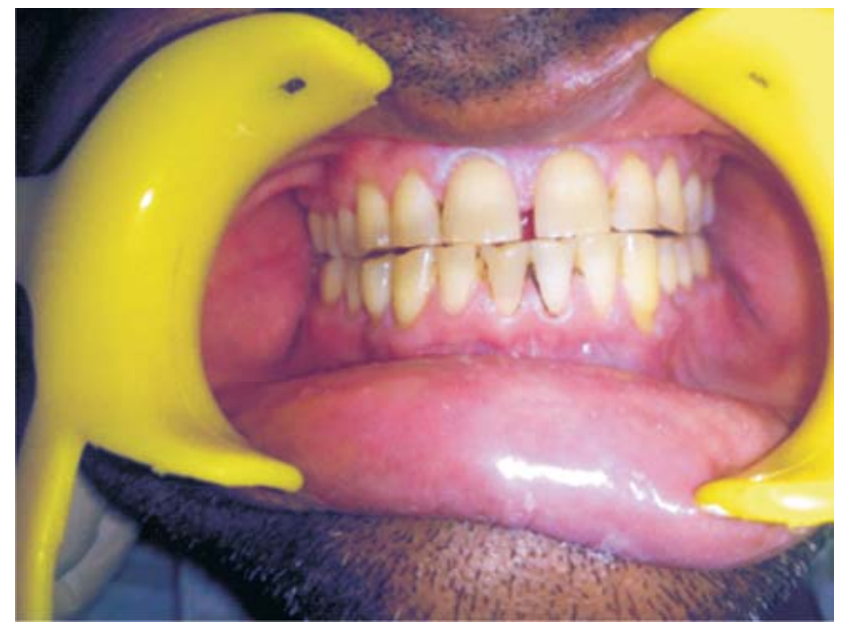

Figure 5: Posterior teeth contacts in protrusive movement

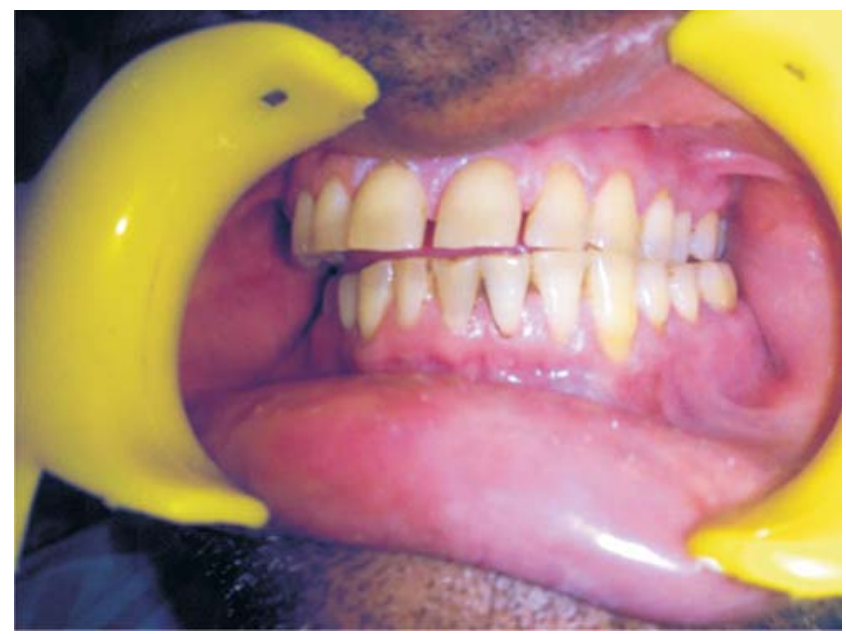

Figure 6: Nonworking contacts in left and right lateral movement

\section{TREATMENT PLAN}

The foremost goal of the treatment plan was to achieve the joint stability by establishing bilateral balanced occlusal contacts along with proper anterior guidance. Stabilization splint was planned at adapted centric relation position. Occlusal splint therapy for joint stabilization and relief of symptoms was given. Follow up visits and required correction of splint several times over 3-4 months was planned. Then the definitive occlusal reconstruction for long term joint stability was to be performed.

Initially, diagnostic casts were fabricated by pouring impressions made with Irreversible hydrocolloid (Zelgan 2002 Densply) and then facebow transfer was done on the semiadjustable articulator (Hanau wide vue ). After deprogramming the jaw muscle engrams by using cotton rolls, a centric interocclusal record as well as protrusive record was made with Aluwax (Aluwax ${ }^{\mathrm{TM}}$ dental bite). This was required to mount the mandibular cast (Figure 7) and record and transfer the condylar guidance on the articulator, respectively. A centric stabilization splint (Figure 8) was fabricated with self activated clear acrylic resin (DPI-RR cold cure)at increased vertical dimension and anterior guidance was established on the bite splint on programmed articulator (Figure 7). The splint was found to be well adapted and stable in mouth and adjusted for uniform posterior occlusal contacts in centric relation position first on articulator and, then in patients mouth. Centric stops were established only on

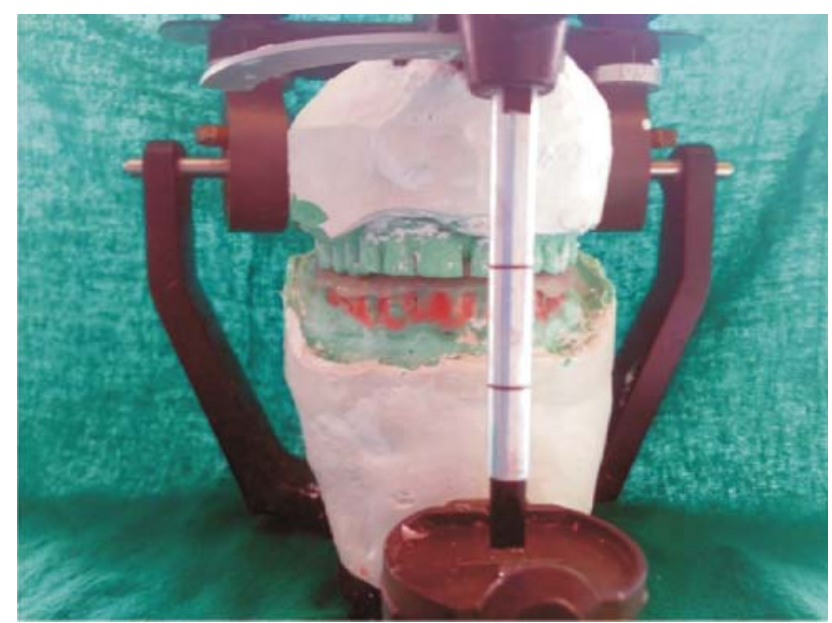

Figure 7: Mounted diagnostic casts and indirect splint fabrication on programmed Hanau Wide Vue 


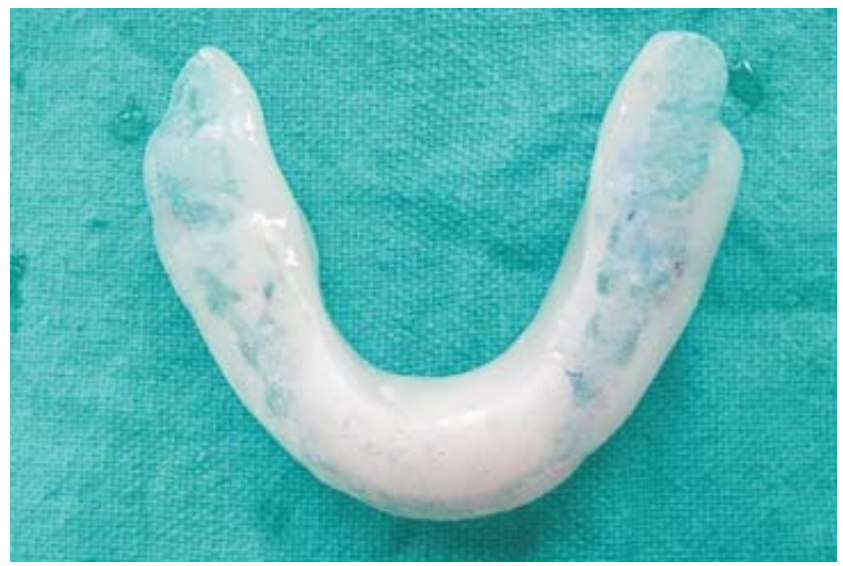

Figure 8: Occlusal splint

functional cusps. Posterior disclusion on protrusion and shallow canine guidance for lateral excursions, was established on the splint (Figure 9).

The patient was instructed to use the splint for maximum number of hours as possible and report next day for any aggravated symptoms. The sign and symptoms were anaylsed according to fonseca's questionnaire $^{3}$ for TMD over 3 days, 1 month, 3 months

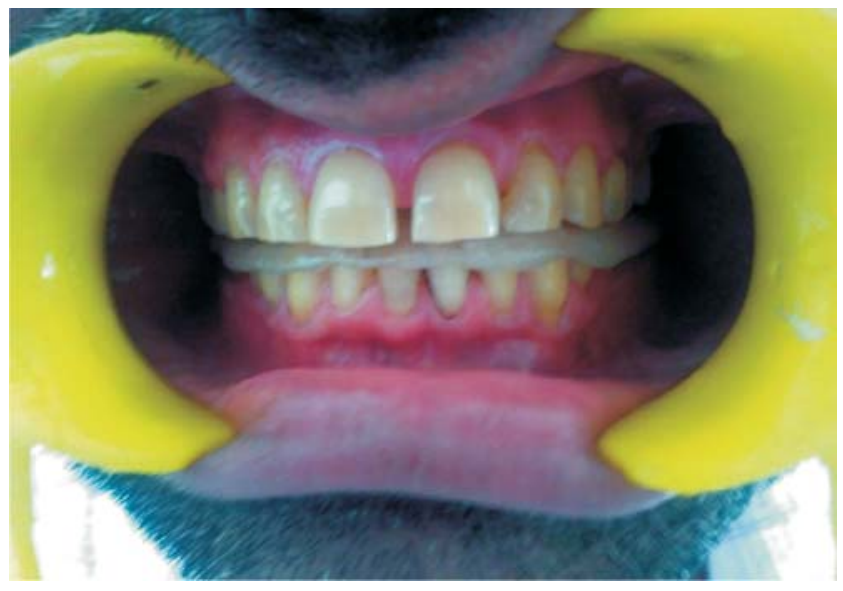

Figure 9: Protrusive and Nonworking contacts in right lateral movement after splint insertion

on scale 0 to 10 . Patient also reported that feeling of right TMJ shifting on opening was not there after wearing of this splint. Patient also did not have to pursue muscle relaxants and analgesics after wearing the splint.

Almost all the symptoms of TMD in the patient got reduced effectively alongwith the progressive reduction of massester muscle mass (Figure 10).
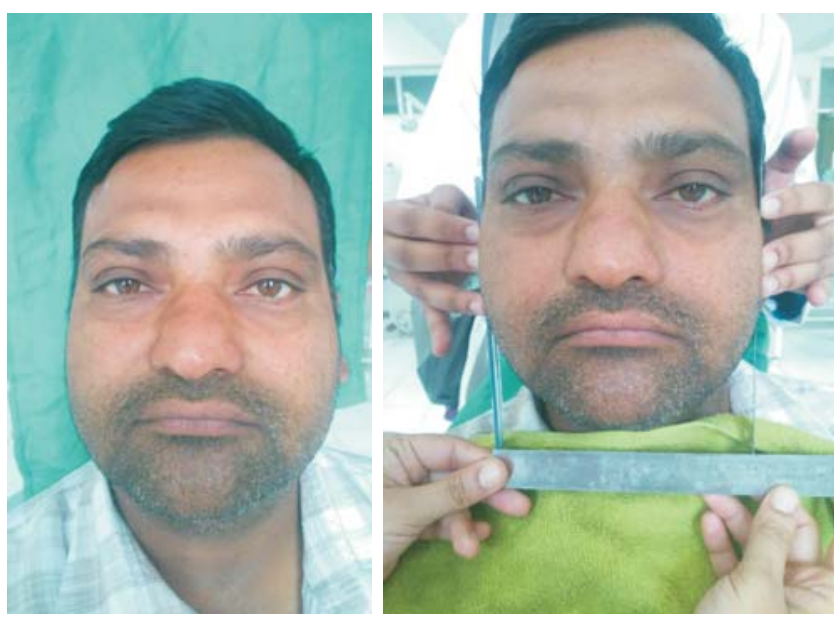

Figure 10: Patients frontal view before and after, showing decreased masseter mass after 3 months of splint wearing

Fonseca 's questionnaire for $\mathrm{TMD}^{3}$

\begin{tabular}{|c|c|c|c|c|}
\hline \begin{tabular}{|c|} 
S. \\
No. \\
\end{tabular} & Questions & \begin{tabular}{|c|} 
Day \\
1
\end{tabular} & $\begin{array}{c}\begin{array}{c}\text { After one } \\
\text { month }\end{array} \\
\end{array}$ & \begin{tabular}{|l|} 
After 3 \\
months \\
\end{tabular} \\
\hline 1. & $\begin{array}{l}\text { Is it hard for you to open } \\
\text { your mouth? }\end{array}$ & 10 & 0 & 0 \\
\hline 2. & $\begin{array}{l}\text { Is it hard for you to move } \\
\text { your mandible from side to } \\
\text { side? }\end{array}$ & 10 & 0 & 0 \\
\hline 3. & $\begin{array}{|lcr|}\text { Do you } & \text { get } & \text { tired/have } \\
\text { muscular } & \text { pain } & \text { while } \\
\text { chewing? } & & \\
\end{array}$ & 10 & 0 & 0 \\
\hline 4. & \begin{tabular}{|l}
$\begin{array}{l}\text { Do you have frequent } \\
\text { headaches? }\end{array}$ \\
\end{tabular} & 10 & 0 & 0 \\
\hline 5. & $\begin{array}{l}\text { Do you have pain on the nape } \\
\text { or stiff neck }\end{array}$ & 0 & 0 & 0 \\
\hline 6. & $\begin{array}{l}\text { Do you have ear aches or } \\
\text { pain in craniomandibular } \\
\text { joints }\end{array}$ & 10 & 4 & 0 \\
\hline 7. & $\begin{array}{l}\text { Have you noticed any TMJ } \\
\text { Clicking while chewing or } \\
\text { when you open your mouth? }\end{array}$ & 0 & 0 & 0 \\
\hline 8. & $\begin{array}{l}\text { Do you clench or grind your } \\
\text { teeth? }\end{array}$ & 10 & 0 & 0 \\
\hline 9. & \begin{tabular}{|l|}
$\begin{array}{l}\text { Do you feel your teeth do not } \\
\text { articulate well }\end{array}$ \\
\end{tabular} & 10 & 10 & 10 \\
\hline 10. & \begin{tabular}{|l|} 
Do you consider yourself a \\
tense (nervous)person?
\end{tabular} & 10 & 8 & 10 \\
\hline & Total score & 70 & 22 & 20 \\
\hline
\end{tabular}

\section{Clinical index classification-Fonseca}

Total between 0 and 15 points

No TMD

Total between 20 and 40 points

Mild TMD

Total between 45 and 65 points

Moderate TMD

Total between 70 and 100 points 
Recall Visit: patient was checked next day after insertion of the prosthesis. All the signs and symptoms were significantly reduced. .There was decrease in symptom of pain within 24 hours. Patient was kept at monthly follow-ups for about four consecutive months. The patient also reported the discontinuation of the analgesics. Reduction in muscle mass of masseter was obvious during each recall visit.

\section{DISCUSSION}

The use of dental occlusal splint followed by permanent occlusal adjustments has been the main stays of TMD treatment. A common goal of occlusal splint treatment is to protect the TMJ Discs from dysfunctional forces a well as to improve the jaw muscle function to relieve associated signs and symptoms by creating harmonious occlusion. ${ }^{4}$ The appliance can be made to cover the occlusal surfaces of maxillary or mandibular teeth and can be fabricated from many different materials, like a hard splint of acrylic resin or soft splint of polyacetamide thermoplastic sheet. ${ }^{5}$ Occlusal splint is beneficial to reduce or eliminate unfavorable loading forces by breaking the muscle engrams and gradually returning the jaw to its most unstrained centric relation position. This slowly alleviates the masticatory muscle pain, TMJ pain, TMJ noises, restricted jaw mobility and all the other signs and symptoms of the TMDs.

One of the important factors that play a role in the longevity and stability of restorative results is the status of tempromandibular joint and neuro musculature system. Bite splint therapy can be effective way to manage signs and symptoms of TMDs both intracapsular and neuromuscular. For the present case, the two goals to be achieved with occlusal splint were;

1. To eliminate/improve patient's symptoms

2. Stability of occlusion with splint for atleast 2-3 months. Splint allows healing, remodelling and adaption of joint structures by controlling forces to the TMJs with properly bite splint design. ${ }^{6}$ Hard acrylic material was chosen for its precise fit and easy to adjust. The mandibular splint was preferred as it was more camouflaged by the lower lip as well as it interferes less during speech.
The splint design has flat posterior surface with only supporting cusp tips touching. These should be preserved to minimize the stimulation of periodontal proprioceptors that provoke the muscular hyperactivity. ${ }^{7}$ This also allowed the condyles to be seated in the most anterosuperior position progressively.

The occlusion developed on the splint varies according to the requirements of the patient. In most instances, multiple occlusal contacts in maximum intercuspation at terminal hinge position of the mandible and in eccentric jaw positions, concept of canine guidance was followed. ${ }^{8}$

Bimanual method of mandibular guidance was used to adjust the splint in centric relation position. Anterior guidance was kept shallow at the same time allowing for posterior disclusion on protrusive movement and right and left canine guidance was established on the working side. The splint was fabricated at the vertical dimensions guided by retruded contact point (i.e just keeping optimum thickness of interocclusal recording medium sufficient enough to keep Retruded Contact Point (RCP) free from occlusion. The incorporation of Curve of Wilson facilitated the working side disclusions by keeping lower lingual cusps slightly shorter than lower buccal cusp. $^{2}$

The shallow anterior guidance on splint also ensured preventing premature anterior contact during clenching which further prevents distalization of condyle and comfort of retrodiscal tissues and that resulted in the reduction of pain.

In this above discussed case, relief for the symptoms was achieved almost overnight. The pain in joint and muscles was reduced and mouth opening improved. The credit for this in major part lies with the patient's self motivation to follow the instructions and his compliance to regular follow ups after the splint therapy.

\section{CONCLUSION}

Over the past 10-20 years, the conceptual basis for using oral appliance in treating TMDs and bruxism has been dramatically redefined. Currently, Occlusal splint 
are still regarded as useful adjuncts for treating certain kinds of TMD patients but the emphasis is entirely on their conservative application. Evidence suggests that Occlusal splint are more effective in treating myogenous problems than intracapsular conditions but can be helpful for both in properly selected cases. There is sufficient literature to support the use of splint therapy to reinstitute neuromuscular harmony in the compromised masticatory system. Dental practitioners have a responsibility to understand and provide this treatment, monitor the condition, and refer the patient to another specialist in the concerned field if necessary.

This case report has highlighted the importance of right diagnosis of the patients problem as well as indirect technique of fabrication of occlusal splint used as therapeutic prosthesis.

The presented case is due for the permanent occlusal adjustments for a definite stable uniform bilateral occlusal relationships which would promote the long term health of the stomatognathic system. The case report to be continued after definite therapy......

\section{REFERENCES}

1. Management of temporomandibular disorders and occlusion.Jeffery P.Okeson.7th edition

2. Functional occlusion from TMJ toSmile Design.Dawson.3rd edition

3. Braz Dent J.2007;18(2)163-7. Use of the Fonseca's Questionaire to assess the prevalence and severity of TMD in Brazilian Dental undergraduates

4. Prasad K, Prasad A : Practical approach to splint therapy. Guident. May2015;16-23

5. Sameh A Seifeldin Khaled A. Elhayes .Soft versus hard occlusal splint therapy in the management of temporomandibular disorders . Saudi Dental Journal $2015 ; 27$ : 208-214.

6. Clinical cases in restorative and reconstructive dentistry edition 1st.Gregory J Tarantole

7. Carlier J.F. Usefulness of splint : J Dentofacial Anom Orthod 2012;15:204

8. Charles A. Kass, and Joseph N. Tregaskes. Occlusal splint fabrication: J Prosthet Dent 1978; 40 : 461-464.

Source of Support: Nil, Conflict of Interest: None Declared 